\title{
Bezerros de Corte Desmamados Precocemente Alimentados com Silagem de Triticale Associada a Diferentes Níveis de Concentrado ${ }^{1}$
}

\author{
Eduardo da Costa Eifert ${ }^{2}$, João Restle ${ }^{3}$, Leonir Luis Pascoal ${ }^{3}$, Ivan Luiz Brondani ${ }^{3}$, Mikael \\ Neumann ${ }^{4}$, José Henrique Souza da Silva ${ }^{3}$, Silvane Barcelos Carlotto ${ }^{5}$
}

\begin{abstract}
RESUMO - Objetivou-se, com este experimento, avaliar o consumo de alimentos, a conversão alimentar e o ganho de peso de bezerros de corte da raça Braford, desmamados aos 88 dias de idade, alimentados com silagem de triticale associada a 35; 45; 55 e $65 \%$ de concentrado, com base na matéria seca. Os animais foram alimentados ad libitum, duas vezes ao dia, com dieta apresentando 15,8\% de proteína bruta. O experimento teve duração de 84 dias, divididos em quatro períodos de 21 dias. Foram avaliados os consumos médios diários de matéria seca (CMS) por animal - em kg (CMSD), por $100 \mathrm{~kg}$ de peso vivo (CMS\%) e por unidade de tamanho metabólico (CMSTM) -, e de fibra em detergente neutro (CFDN) - em kg e por $100 \mathrm{~kg}$ de peso vivo (CFDN\%) - a conversão alimentar (CA) e o ganho de peso médio diário (GMD). A análise de regressão apresentou respostas lineares e crescentes para os níveis de concentrado nas variáveis CMSD, CMS\%, CMSTM e GMD. O GMD foi de 270, 536, 728 e 784 g, respectivamente, para os quatro níveis de concentrado. O componente quadrático foi observado para CFDN, CFDN\%, CA e peso final. Não houve interação entre período e nível de concentrado para as variáveis estudadas, porém o CMS, CMS\%, CFDN\% e GMD aumentaram linearmente com o avanço do período experimental. Para obter taxas de ganho de peso adequadas visando o desmame precoce, recomenda-se associar à silagem de triticale $45 \%$, ou mais, de concentrado.
\end{abstract}

Palavras-chave: confinamento, conversão alimentar, desmame precoce, ganho de peso, idade de desmame

\section{Performance of Beef Calves Weaned at 88 Days Old Fed Triticale Silage and Different Concentrate Levels}

\begin{abstract}
The objective of this experiment was to evaluate the food intake, food conversion and weight gain of Braford calves, weaned with 88 days of age, fed with triticale silage associated to $35,45,55$ or $65 \%$ concentrate, dry matter basis. The animals were fed ad libitum, twice daily with the $15 \% \mathrm{CP}$ diet. The experiment was conducted during 84 days, divided in four periods of 21 days. Average daily intakes of dry matter (DMI) expressed in $\mathrm{kg}$ (DMID), per $100 \mathrm{~kg}$ of live weight (DMI\%) and per unit of metabolic weight (DMIMW), of NDF in $\mathrm{kg}$ (NDFI) and per $100 \mathrm{~kg}$ of live weight (NDFI\%), food conversion (FC) and average daily weight gain (ADG) were evaluated. The regression analysis showed linear increases for DMID, DMI\%, DMIMW and ADG, as the concentrate level increased. The ADG was 270, 536, 728 and $784 \mathrm{~g}$, respectively, for the four concentrate levels. Quadratic answers were observed for NDFI, NDFI\%, final weight and FC, as the concentrate level increased. No interaction was observed between concentrate level and experimental period for the studied variables, but DMID, DMI\%, NDFI\% and ADG increased as the experimental period advanced. To obtain rates of weight gain appropriate, aiming the early weaning, it is recommended to associate the triticale silage with $45 \%$ or more concentrate in the diet.
\end{abstract}

Key Words: confinement, early weaning, food conversion, weaning age, weight gain

\section{Introdução}

O campo nativo é a base forrageira para a produção de bovinos de corte no sul do Brasil e caracteriza-se por apresentar produção estival, com grande variação em relação à sua produção e qualidade nutritiva. Esta base forrageira permite produções adequadas em condições extensivas de exploração, entretanto, apresenta limitações quando se intensifica o sistema. Quanto aos rebanhos de cria, as variações na oferta e qualidade forrageira não permitem que se obtenham vacas com adequado estado corporal durante a estação de parição e o período de acasalamento, refletindo em taxas de natalidade pouco acima de 50\% (ANUALPEC, 2003).

Sabe-se que os níveis nutricionais nos períodos pré e pós-parto apresentam forte influência sobre a repetição de cria (Wiltbank et al., 1962). A utilização de pastagens cultivadas de inverno, como a aveia e o azevém (Costa et al., 1981), ou a suplementação com

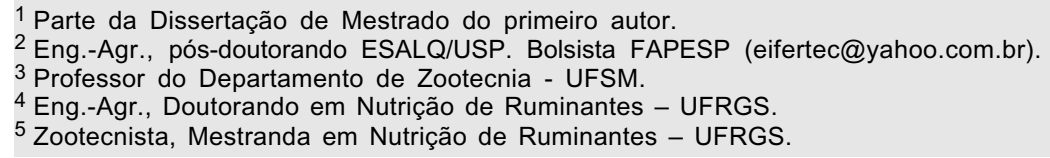


concentrados na alimentação destes animais têm apresentado respostas positivas. No entanto, o alto custo de produção e o longo período de retorno desestimulam o produtor rural a adotar estas tecnologias. O desmame precoce, realizado quando os bezerros atingem 60 a 90 dias de idade, é uma prática adotada com sucesso e bastante utilizada pelos produtores rurais, com o objetivo de aumentar os índices reprodutivos do rebanho de cria a um custo compatível com o sistema de exploração pecuária (Pascoal et al., 2000).

$\mathrm{O}$ desmame realizado nesta faixa etária permite que a vaca alcance maior ganho de peso, menor intervalo de partos e melhor estado corporal que as vacas com bezerro ao pé. A interrupção antecipada da lactação garante que a vaca direcione os nutrientes ingeridos para a recomposição corporal, involução uterina e reprodução. Observa-se, portanto, maior taxa de prenhês para as vacas que tiveram seus bezerros desmamados precocemente, com maior magnitude quanto menor a idade do bezerro (Moletta \& Perotto, 1997), para novilhas em crescimento, vacas de primeira cria (Cerdótes et al., 2004) e para aquelas com baixa condição corporal (Bishop et al., 1994) ou quando há baixa oferta de alimento (Moojen et al., 1994; Simeone \& Lobato, 1996).

A principal limitação na prática do desmame precoce é em relação à alimentação do bezerro, que depende do leite para atender às suas necessidades nutricionais de crescimento. Bezerros alimentados somente com pastagens na fase de desmame têm apresentado crescimento inferior aos desmamados aos sete meses, em razão da baixa capacidade de ingestão, embora Muhelmann et al. (1997) tenham observado crescimento compensatório quando a alimentação subseqüente apresentou alta qualidade. Portanto, a utilização de concentrados torna-se necessária para a complementação das dietas destes animais.

Para bezerros desmamados precocemente, torna-se interessante buscar o rápido desenvolvimento dos compartimentos gastrintestinais, uma vez que o animal passa a consumir unicamente alimentos sólidos. Thomas \& Tinnimit (1976) observaram que proteína bruta e consumo foram igualmente importantes na fase de 41 a 72 dias e, após, o consumo de matéria seca foi mais importante que a porcentagem de proteína na dieta. Para bezerros desmamados com idades a partir de 66 dias consumindo $50 \%$ de concentrado na dieta, não tem sido observada diferença no ganho de peso ou no consumo, quando os níveis de proteína bruta variaram de 13 a 19 (Pascoal et al., 2000) e de 14 a 20\% na dieta (Restle et al., 2001).
Quando se objetiva o abate aos 24 meses de idade, as taxas de ganho de peso pretendidas para esta fase ficam entre 0,500 e $0,600 \mathrm{~kg} / \mathrm{dia}$, que têm sido alcançadas com a suplementação de $1 \%$ do peso vivo do animal com concentrado. No entanto, são raras as publicações no país que avaliam o consumo e a resposta produtiva de bezerros desmamados precocemente alimentados com diferentes níveis de concentrado.

Sabendo-se dos efeitos da elevação da densidade da dieta sobre os fatores que regulam o consumo (Mertens, 1994), e considerando o consumo a variável de maior influência sobre o ganho de peso, torna-se necessário quantificar a resposta desta categoria a crescentes níveis de concentrado, possibilitando traçar recomendações para a prática do desmame precoce, como pretendeu-se neste trabalho.

\section{Material e Métodos}

O trabalho foi conduzido no Departamento de Zootecnia da Universidade Federal de Santa Maria, entre os meses de janeiro e maio de 1998. Foram utilizados 24 bezerros inteiros, da raça Braford (5/8 Hereford 3/8 Nelore), desmamados com 88 dias de idade e $96,2 \mathrm{~kg}$ de peso médio.

Os tratamentos consistiram da associação da silagem de triticale (Xtriticossecale) com os níveis de concentrado de $35 ; 45 ; 55$ e $65 \%$ e foram dispostos em delineamento experimental inteiramente casualizado, com quatro tratamentos e duas repetições, sendo cada uma formada por um lote de três bezerros.

Os níveis de $\mathrm{PB}$ das dietas foram calculados conforme a proporção dos ingredientes para os diferentes níveis de concentrado de cada tratamento $(15,8 \% \mathrm{em}$ média). O concentrado foi constituído por farelo de soja (92,6\% DIVMO; 94,2\% MO; 50,9\% PB; 14,9\% FDN), grão de sorgo triturado (84,0\% DIVMO; 99,1\% MO; 9,0\% PB; 16,1\% FDN), sal comum, calcário calcítico, fosfato bicálcico e ionóforo. Para confecção da silagem, o triticale foi colhido em fase de grão leitoso, com tamanho de picado mínimo de $30 \mathrm{~mm}$, cortado rente ao solo e armazenado em silo 'aéreo' (26,3\% MS; 93,1\% MO; 52,6\% DIVMO; 11,1\% PB; 65,6\% FDN). Os resultados das análises bromatológicas das dietas são apresentados na Tabela 1.

A fase de adaptação ao manejo e à alimentação teve duração de 21 dias, desde o desmame até o início do experimento. Durante os primeiros dez dias desta fase, os animais receberam concentrado comercial (24\% PB) em quantidade equivalente a $1,2 \%$ do peso 
vivo e capim-elefante verde picado, até que todos apresentassem consumo regular. Nos onze dias seguintes, os animais foram pesados, sorteados e, gradativamente, adaptados à silagem de triticale e aos níveis de concentrado a que seriam submetidos.

Os animais foram alojados em oito piquetes com $40 \mathrm{~m}^{2}$ de área, providos de bebedouro regulado por bóia automática e cocho de madeira com disponibilidade de $0,70 \mathrm{~m}$ para cada animal. A dieta foi fornecida ad libitum, em partes iguais, duas vezes ao dia, sendo as sobras retiradas do cocho e pesadas antes da alimentação matutina. O período experimental foi de 84 dias e, para controle da evolução do peso, foram realizadas pesagens periódicas a cada 21 dias, com jejum prévio de sólidos de 14 horas.

As variáveis estimadas foram os consumos médios diários por animal de matéria seca (CMS), expresso em $\mathrm{kg}$ (CMSD), por $100 \mathrm{~kg}$ de peso vivo (CMS\%), por unidade de tamanho metabólico (CMSTM), de

Tabela 1 -Porcentagem de concentrado, silagem e composição bromatológica das dietas experimentais

Table 1 - Concentrate and silage percentages and chemical composition of the experimental diets

\begin{tabular}{|c|c|c|c|c|}
\hline \multirow[t]{2}{*}{$\begin{array}{l}\text { Ingrediente } \\
\text { Ingredient }\end{array}$} & \multicolumn{4}{|c|}{$\begin{array}{c}\text { Nível de concentrado, } \% \\
\text { Concentrate level, \% }\end{array}$} \\
\hline & 35 & 45 & 55 & 65 \\
\hline & \multicolumn{4}{|c|}{$\begin{array}{c}\text { Composição percentual, \% MS } \\
\text { Percent composition, } \% D M\end{array}$} \\
\hline $\begin{array}{l}\text { Silagem de triticale, } \% \\
\text { Triticale silage, \% }\end{array}$ & 65,28 & 55,16 & 43,98 & 35,09 \\
\hline $\begin{array}{l}\text { Concentrado, } \% \\
\text { Concentrate, \% } \\
\text { Composição bomatológ } \\
\text { Chemical composition, }\end{array}$ & 34,72 & 44,84 & 56,03 & 64,90 \\
\hline $\begin{array}{l}\text { MS, \% } \\
D M, \%\end{array}$ & 46,38 & 52,41 & 59,08 & 64,83 \\
\hline $\begin{array}{l}\mathrm{PB}, \% \\
C P, \%\end{array}$ & 15,60 & 15,72 & 15,93 & 15,95 \\
\hline $\begin{array}{l}\text { FDN, } \% \\
N D F, \%\end{array}$ & 47,97 & 42,91 & 37,32 & 32,97 \\
\hline $\begin{array}{l}\mathrm{FDA}, \% \\
A D F, \%\end{array}$ & 20,68 & 18,87 & 16,87 & 15,34 \\
\hline $\begin{array}{l}\mathrm{ED}, \mathrm{Mcal} / \mathrm{kg}^{1} \\
\text { DE, } \text { Mcal } / \mathrm{kg}\end{array}$ & 3,028 & 3,121 & 3,224 & 3,325 \\
\hline $\begin{array}{l}\mathrm{ELm}, \mathrm{Mcal} / \mathrm{kg}^{2} \\
\mathrm{NEm}, \mathrm{Mcal} / \mathrm{kg}\end{array}$ & 1,592 & 1,658 & 1,731 & 1,819 \\
\hline $\begin{array}{l}\mathrm{ELg}, \mathrm{Mcal} / \mathrm{kg}^{2} \\
N E g, M c a l / k g\end{array}$ & 0,990 & 1,049 & 1,113 & 1,175 \\
\hline
\end{tabular}

R. Bras. Zootec., v.33, n.6, p.1806-1813, 2004 (Supl. 1)
FDN em $\mathrm{kg}(\mathrm{CFDN})$ e por $100 \mathrm{~kg}$ de peso vivo (CFDN\%), a conversão alimentar (CA) e o ganho de peso médio diário (GMD). Para o cálculo da energia digestível das dietas, foram utilizadas as equações sugeridas pelo ARC (1980), com base na DIVMO e na matéria orgânica ( $\mathrm{MO}, \%)$. A partir desta, o valor de ED foi multiplicado por 0,82, para se estimar a energia metabolizável (EM), calculando-se a energia líquida de mantença (ELm) e de ganho (ELg) de acordo com as equações sugeridas pelo NRC (1984).

As variáveis acima descritas foram avaliadas considerando o período experimental total e cada intervalo das pesagens ( 21 dias), a fim de avaliar a evolução do crescimento e o consumo alimentar dos bezerros. Assumiu-se o CMS\% e o GMD como dados não-contínuos para verificar a possível interação entre o nível de concentrado e o período experimental.

Os resultados obtidos foram submetidos à análise de variância e de regressão linear, com o auxílio dos comandos 'Proc GLM' e 'Proc Reg' do programa estatístico SAS (SAS, 1990). Para a escolha dos modelos de regressão polinomial que melhor ajustaram os dados de cada variável dependente, foram usados os testes dos coeficientes de regressão $\left(\mathrm{H}_{0}: \hat{a}_{\mathrm{K}}=0\right)$ linear e quadrático. Para a normatização dos dados, o valor de todas as variáveis estudadas foi transformado para sua raiz quadrada, antes da análise estatística.

\section{Resultados e Discussão}

A análise bromatológica das dietas (Tabela 1) comprovou que as relações volumoso:concentrado dos tratamentos foram pouco diferentes dos níveis de concentrado $(35,45,55$ e $65 \%)$. Embora os dados da análise bromatológica tenham sido utilizados na análise estatística, convencionou-se empregar os valores de concentrado para referenciar os tratamentos na discussão deste trabalho.

$\mathrm{Na}$ Tabela 2, são apresentados os valores médios e as equações de regressão para o consumo de matéria seca e de FDN, expressos nas diferentes formas. $\mathrm{O}$ consumo de matéria seca diário aumentou linearmente à medida que se elevou o nível de concentrado na dieta, variando de 2,24 a 3,87 kg/dia. Quando o consumo de matéria seca foi expresso em relação a $100 \mathrm{~kg}$ de peso vivo ou por unidade de tamanho metabólico, o incremento também foi linear, de 27,8 g e 1,39 g para cada nível de concentrado na dieta, respectivamente. 
Os efeitos lineares e positivos verificados para CMS\% e CMSTM com a inclusão de concentrado na dieta podem ser explicados pelo aumento na densidade física da dieta, pela diminuição do tamanho da partícula e pelos reflexos na velocidade de passagem (Desbordes et al., 1984). Segundo Mertens (1994), a variação no consumo de matéria seca segue distribuição de forma quadrática, com aumentos nos níveis de concentrado e representam os controles físicos e quimiostáticos, como observado por Araújo et al. (1998), ao aumentarem o nível de concentrado 25 para $75 \%$ na alimentação de bezerros leiteiros em confinamento. No presente trabalho, verificaram-se respostas lineares no CMS até o nível $65 \%$ de concentrado, o que impediu a estimativa do nível de concentrado que permitisse atingir o máximo consumo desta categoria.

O CFDN e CFDN\% apresentaram respostas quadráticas ao incremento de concentrado, com valores máximos estimados para os níveis de 54,5 e $57,25 \%$ de concentrado na dieta, respectivamente. Os valores observados para CFDN\%, com média de $0,944 \mathrm{~kg} / 100 \mathrm{~kg} \mathrm{PV}$, estão próximos aos verificados por outros autores para bezerros desmamados precocemente. Com bezerros de mesmo grupo genético ao do presente trabalho, desmamados a partir dos 66 dias de idade e alimentados com silagem de aveia e $50 \%$ de concentrado, Pascoal et al. (2000) e Pascoal et al. (2001a) observaram CFDN\% de 1,032 e 0,793 $\mathrm{kg} / 100 \mathrm{~kg}$ PV, respectivamente.

Mertens (1994) sugere que o CMS de vacas de leite é maximizado quando o CFDN\% é próximo a
$1,2 \mathrm{~kg} \mathrm{FDN} / 100 \mathrm{~kg}$ PV e para dietas que proporcionem valores inferiores, o consumo teria um controle fisiológico, pois atenderia aos requerimentos energéticos dos animais antes do efeito de enchimento. Embora tenham sido verificados valores de CFDN\% abaixo de $1,2 \%, \mathrm{o}$ CMSD dos bezerros possivelmente foi limitado pela capacidade física de ingestão. A FDN é a porção do alimento que mais ocupa volume e de mais lenta digestão (Allen, 1996) e estes animais, no início do confinamento, não apresentavam a máxima capacidade de distensão ruminal. Ainda, bezerros apresentam menores efetividade e freqüência de mastigação que animais adultos (Welch, 1982), o que influencia a quebra das partículas fibrosas, justificando o menor consumo nos níveis mais baixos de concentrado.

O CMSD e CMS\% verificados neste trabalho estão próximos aos observados por outros autores, ao avaliarem o desempenho de bezerros desmamados precocemente alimentados com silagem de cereais de inverno. Trabalhando com silagem de aveia e com $50 \%$ de concentrado, Pascoal et al. (2000) e Pascoal et al. (2001a) observaram CMS\% de 2,5 e $2,4 \%$, respectivamente, valores muito próximos aos verificados no nível de $55 \%$.

Quando se utiliza silagem de sorgo como fonte de volumoso, os animais desta categoria apresentam maiores valores para o CMSD. Restle et al. (2001) e Pascoal et al. (2001b) reportam CMS\% próximos a $3,0 \%$ do PV e Eifert et al. (1999) observaram respostas lineares ao incremento de concentrado, com média de $\mathrm{CMS} \%$ de $2,61 \%$. Apesar de as diferenças

Tabela 2 - Valores médios e equações de regressão do consumo de matéria seca diário (CMSD), por $100 \mathrm{~kg}$ de peso vivo (CMS\%) e em relação ao tamanho metabólico (CMSTM), consumo de FDN diário (CFDN) e consumo de FDN por $100 \mathrm{~kg}$ de peso vivo (CFDN\%), de acordo os níveis de concentrado

Table 2 - Means and regression equations for daily dry matter intake (DMID), per $100 \mathrm{~kg}$ of body weight (DMI\%) and metabolic weight (DMITM), NDF daily intake (NDFI) and per $100 \mathrm{~kg}$ of body weight (NDFI\%), according to concentrate level

\begin{tabular}{|c|c|c|c|c|c|}
\hline \multirow[t]{2}{*}{$\begin{array}{l}\text { Variável } \\
\text { Variable }\end{array}$} & \multicolumn{4}{|c|}{$\begin{array}{c}\text { Nível de concentrado } \\
\text { Concentrate level }\end{array}$} & \multirow[t]{2}{*}{$\begin{array}{l}\text { Equação de regressão } \\
\text { Regression equation }\end{array}$} \\
\hline & 35 & 45 & 55 & 65 & \\
\hline CMS, kg/dia (DMI, kg/day) & 2,24 & 3,01 & 3,49 & 3,87 & ${ }^{1} \hat{\mathrm{Y}}=2,7339+0,0188 \mathrm{NC}$ \\
\hline CMSD $\%, \mathrm{~kg} / 100 \mathrm{~kg} P V(D M I D \%, \mathrm{~kg} / 100 \mathrm{~kg} P V)$ & 1,97 & 2,36 & 2,59 & 2,83 & ${ }^{2} \hat{\mathrm{Y}}=1,0392+0,0280 \mathrm{NC}$ \\
\hline CMSTM, kg/PV ${ }^{0,75}\left(D M I M W, k g / P V .^{75}\right)$ & 0,085 & 0,104 & 0,116 & 0,127 & ${ }^{3} \hat{\mathrm{Y}}=0,0386+0,0014 \mathrm{NC}$ \\
\hline CFDN, kg/dia (NDFI, kg/day) & 1,056 & 1,265 & 1,275 & 1,246 & ${ }^{4} \hat{\mathrm{Y}}=-0,4973+0,0654 \mathrm{NC}-0,0006 \mathrm{NC}^{2}$ \\
\hline CFDN $\%, \mathrm{~kg} / 100 \mathrm{~kg}$ PV $(N D F I \%, \mathrm{~kg} / 100 \mathrm{~kg} P V)$ & 0,930 & 0,990 & 0,946 & 0,910 & ${ }^{5} \hat{\mathrm{Y}}=0,4286+0,0229 \mathrm{NC}-0,0002 \mathrm{NC}^{2}$ \\
\hline
\end{tabular}

${ }^{1} \mathrm{r}^{2}=0,2068 ; \mathrm{CV}=11,54 \% ; \mathrm{P}<0,0089$.

${ }^{2} \mathrm{r}^{2}=0,7916 ; \mathrm{CV}=6,80 \% ; \mathrm{P}<0,0001$

${ }^{3} \mathrm{r}^{2}=0,7931 ; \mathrm{CV}=7,58 \% ; \mathrm{P}<0,0001$

${ }^{4} r^{2}=0,3937 ; C V=9,50 ; P<0,0064$.

$5 r^{2}=0,1678 ; C V=6,60 ; P<0,0382$

$\mathrm{NC}$ - nível de concentrado (NC - concentrate level).

R. Bras. Zootec., v.33, n.6, p.1806-1813, 2004 (Supl. 1) 
no CMSD dos experimentos supracitados estarem relacionadas ao grupo genético dos animais, ao peso e à idade ao desmame e às características inerentes às dietas, deve-se considerar que as forragens de cereais de inverno são colhidas com maior umidade que as de sorgo e de milho e dependentes dos carboidratos solúveis para adequada fermentação, podendo alterar negativamente a palatabilidade e o odor da silagem.

O GMD variou de 270 para $784 \mathrm{~g}$, respectivamente, para os níveis 35 e $65 \%$ de concentrado (Tabela 3 ). Da mesma forma que para o CMSD, observou-se, com o incremento nos níveis de concentrado, regressão linear e positiva do GMD, de 17,3 g para cada unidade de concentrado na dieta (Tabela 3). Araújo et al. (1998) constataram que a resposta do GMD, em função do nível de concentrado, é curvilínea e nãolinear, concordando com os resultados obtidos por Eifert et al. (1999), com bezerros de mesma categoria e grupo genético do presente trabalho. Neste último estudo, níveis de 35 a $65 \%$ de concentrado foram associados à silagem de sorgo e o maior GMD estimado foi obtido para dietas com $56,1 \%$ de concentrado. Segundo esses autores, redução na taxa de ganho de peso nos níveis mais altos de concentrado estaria relacionada à indução à engorda precoce e aos efeitos negativos sobre o ambiente ruminal, envolvendo a diminuição do $\mathrm{pH}$, degradação da fibra e acidose subclínica.

A conversão alimentar também apresentou distribuição quadrática, com melhor conversão calculada de 4,85 para o nível de $58,7 \%$ de concentrado. De acordo com Restle \& Vaz (1998), a conversão ali- mentar desta categoria situa-se na faixa de 4,0 a 5,0 , o que representa a eficiência com que os nutrientes da dieta são absorvidos e transformados em ganho de peso, posto que, neste estádio, ocorre principalmente crescimento muscular.

O tratamento $45 \%$ proporcionou consumo de concentrado de $0,96 \%$ do peso vivo, bastante próximo ao nível de suplementação utilizado para desmamar animais em pastagens, de 1\% PV (Restle \& Vaz, 1998) (Tabela 3). O GMD obtido com este nível de concentrado foi de $536 \mathrm{~g}$, próximo ao relatado por Moletta \& Perotto (1997), que desmamaram bezerros em pastagem de capim-hermátria aos 70 dias de idade e o grupo suplementado com $1 \%$ do PV apresentou ganho de $518 \mathrm{~g} / \mathrm{dia}$, similar aos animais que permaneceram ao pé da vaca até os 210 dias $(556 \mathrm{~g} / \mathrm{dia})$.

Pascoal et al. (1999) simularam várias dietas para o desmame em confinamento ou em pastagens, com dados baseados na literatura, e observaram que a suplementação com $1 \%$ do peso vivo é suficiente para ganhos de peso de até $0,500 \mathrm{~kg} / \mathrm{dia}$, mas, para ganhos maiores que $0,600 \mathrm{~kg} / \mathrm{dia}$, o nível de suplementação deve ser aumentado para 1,25\%. Confirmando estas afirmações (Tabela 5) os tratamentos 55 e $65 \%$ equivaleram a consumos de concentrado de 1,46 e $1,86 \%$ do PV, correspondendo a ganhos próximos de $750 \mathrm{~g} /$ dia.

Tem-se verificado que os ganhos de peso de bezerros desmamados aos 210 dias variam de $500 \mathrm{a} 600 \mathrm{~g} / \mathrm{dia}$ (Costa et al., 1981; Ribeiro \& Restle, 1991; Moletta \& Perotto, 1997) Os ganhos obtidos com os diferentes níveis de concentrado utilizados neste trabalho condizem com o objetivo do desmame precoce, com exceção

Tabela 3 - Valores médios e equações de regressão do peso inicial (PI) e final (PF), ganho médio diário (GMD), conversão alimentar (CA) e consumo de concentrado por $100 \mathrm{~kg}$ de peso vivo (CCP), de acordo os níveis de concentrado

Table 3 - Means and regression equations for initial (IW) and final weight (FW), average daily gain (ADG), food conversion (FC) and concentrate intake per $100 \mathrm{~kg}$ of body weight (CIP), according to the concentrate level

\begin{tabular}{lccccc}
\hline Variável & \multicolumn{4}{c}{$\begin{array}{c}\text { Nível de concentrado } \\
\text { Concentrate level }\end{array}$} & $\begin{array}{c}\text { Equação de regressão } \\
\text { Regression equation }\end{array}$ \\
\cline { 2 - 5 } & 35 & 45 & 55 & 65 & $\hat{\mathrm{Y}}=105,0$ \\
$\mathrm{PI}, \mathrm{kg}(\mathrm{IW}, \mathrm{kg})$ & 102,5 & 106,7 & 105,5 & 105,2 & $2 \hat{\mathrm{Y}}=-54,2396+7,0667 \mathrm{NC}-0,0554 \mathrm{NC} C^{2}$ \\
$\mathrm{PF}, \mathrm{kg}(\mathrm{FW}, \mathrm{kg})$ & 125,2 & 151,7 & 166,7 & 171,0 & $3 \hat{\mathrm{Y}}=-0,2877+0,017341 \mathrm{NC}$ \\
$\mathrm{GMD}, \mathrm{kg} / \mathrm{dia}(A D G, \mathrm{~kg} /$ day $)$ & 0,270 & 0,536 & 0,728 & 0,784 & $4 \hat{\mathrm{Y}}=31,4236-0,9047 \mathrm{NC}+0,0077 \mathrm{NC} C^{2}$ \\
$\mathrm{CA}(F C)$ & 9,17 & 6,41 & 4,90 & 5,22 & $\hat{\mathrm{Y}}=1,24$ \\
$\mathrm{CCP}, \%(C I P, \%)$ & 0,71 & 0,96 & 1,46 & 1,83 & \\
\hline
\end{tabular}

${ }^{2} \mathrm{r}^{2}=0,9360 ; \quad P<0,0001$.

${ }^{5} \mathrm{r}^{2}=0,5732 ; \quad P<0,0078$

$3 \mathrm{r}^{2}=0,7939 ; ; \mathrm{P}<0,0001$.

$\mathrm{NC}$ - nível de concentrado (NC - concentrate level).

R. Bras. Zootec., v.33, n.6, p.1806-1813, 2004 (Supl. 1) 
do tratamento $35 \%$. Os pesos finais observados para os tratamentos 45,55 e $65 \%$ de concentrado, próximos a $160 \mathrm{~kg}$ aos seis meses, permitem projetar que o crescimento ponderal destes animais é adequado para o abate aos 24 meses de idade.

O menor desempenho dos animais no tratamento $35 \%$ reflete a necessidade energética desta categoria; a menor densidade energética das dietas destes animais, associada ao efeito de enchimento ruminal da dieta e à necessidade de desenvolvimento ruminal, pode ter limitado o ganho de peso pelo aumento da necessidade de energia de mantença. Dessa forma, torna-se questionável a recomendação de níveis de concentrado abaixo de $45 \%$ ou $1 \%$ do peso vivo.

Constam, na Tabela 4, os consumos de energia líquida de mantença e de ganho (CELm e CELg), o consumo de proteína (CPB) e o GMD observados e comparados com as equações sugeridas pelo NRC (1984), representados pelo balanço de energia líquida consumida (CELg) e balanço de proteína bruta consumida (BPB). O consumo de PB foi bastante próximo e pouco superior aos recomendados em todos os tratamentos (45 g/dia, em média). O CELg foi superior aos valores sugeridos pelas equações do NRC (1984) para as taxas de ganhos observadas em todos os tratamentos. As maiores diferenças entre os valores observados e os calculados pelas equações referem-se aos tratamentos com menores quantidades de concentrado, apresentando um balanço de $+0,440$ e $+0,307 \mathrm{Mcal} /$ dia para os tratamentos $35 \mathrm{e}$ $45 \%$, respectivamente.
Quando os valores do CELg e CPB foram adicionados nas equações do NRC (1984), as taxas de ganho estimadas foram superiores às observadas, embora tenha ocorrido aproximação nos níveis de 55 e $65 \%$ de concentrado. Pascoal et al. (2000), trabalhando com bezerros Braford desmamados aos 66 dias de idade e alimentados com silagem de aveia e $50 \%$ de concentrado, verificaram que o CELg dos animais não alcançou os valores sugeridos pelo NRC (1984).

Possivelmente, estas diferenças estejam relacionadas com a metodologia de estimativa do valor energético dos alimentos. Nesse sentido, Rocha Jr. et al. (2003) observaram que os valores para FDN digestível de alimentos volumosos tropicais foram subestimados de acordo com as equações do NRC (2001). Por outro lado, o NRC (1984) ainda não dispunha em suas estimativas a correção da digestibilidade de acordo com o nível de consumo, presente no NRC (1996).

A maior taxa de ganho de peso estimada pelas equações pode estar relacionada com a categoria animal avaliada, pois o NRC (1984) não computa a fase desenvolvimento da capacidade de ingestão dos bezerros. Neste sentido, Pascoal et al. (2000) observaram que o consumo de bezerros provenientes do desmame precoce aumentou linearmente com o avanço do período experimental e os consumos de PB elevaram de 349 para $570 \mathrm{~g} /$ dia desde o início até os 84 dias do período experimental.

Consta, na Figura 1, a evolução do CMS\% (1A) e do GMD (1B) dos animais com o avanço do período

Tabela 4 - Estimativas do consumo de energia líquida de mantença (CElm), energia líquida de ganho (CElg), e de proteína bruta (CPB), balanço de ELg e de PB consumidas (BELg e BPB), comparada com as propostas pelas equações do NRC (1984) para os ganhos de peso obtidos (GMD)

Table 4 - Estimates for consumed net energy of maintenance (CNEm, Mcal/day), net energy for gain (CNEg, Mcal/day), intake of crude protein (CP, g/day), CNEg and CP balance (BNEg and BCP), compared to the equations proposed by equations of the NRC (1984) for the weight gains obtained (ADG, $\mathrm{kg} / \mathrm{dia}$ )

\begin{tabular}{|c|c|c|c|c|}
\hline \multirow[t]{2}{*}{$\begin{array}{l}\text { Variável } \\
\text { Variable }\end{array}$} & \multicolumn{4}{|c|}{$\begin{array}{l}\text { Nível de concentrado, \% } \\
\text { Concentrate level, \% }\end{array}$} \\
\hline & 35 & 45 & 55 & 65 \\
\hline CELm NRC, Mcal/dia (CNEm NRC, Mcal/day) & 2,684 & 2,951 & 3,068 & 3,102 \\
\hline CELg NRC, Mcal/dia (CNEg NRC, Mcal/day) & 0,462 & 1,076 & 1,568 & 1,717 \\
\hline CELg obs., Mcal/dia (CNEg obs., Mcal/day) & 0,902 & 1,383 & 1,693 & 1,881 \\
\hline BELg, Mcal/dia (BNEg, Mcal/day) & 0,440 & 0,307 & 0,125 & 0,164 \\
\hline CPB NRC, $\mathrm{kg} / \mathrm{dia}$ (CPINRC, $\mathrm{kg} /$ day) & 0,297 & 0,441 & 0,529 & 0,565 \\
\hline CPB obs., kg/dia (CPI obs., kg/day) & 0,354 & 0,479 & 0,561 & 0,622 \\
\hline $\mathrm{BPB}, \mathrm{kg} / \mathrm{dia}(B C P, \mathrm{~kg} /$ day $)$ & 0,057 & 0,038 & 0,032 & 0,057 \\
\hline GMD NRC, $\mathrm{kg} / \mathrm{dia}(A D G N R C, \mathrm{~kg} /$ day $)$ & 0,497 & 0,673 & 0,781 & 0,851 \\
\hline GMD obs., kg/dia (ADG obs., kg/day) & 0,270 & 0,536 & 0,729 & 0,783 \\
\hline
\end{tabular}

R. Bras. Zootec., v.33, n.6, p.1806-1813, 2004 (Supl. 1) 
experimental. Não foi verificada interação significativa $(\mathrm{P}>0,05)$ entre período e nível de concentrado representado pelas equações: $\mathrm{MS} \%=2,0595+0,0090 \mathrm{P}$ $\left(\mathrm{r}^{2}=0,2625 ; \quad \mathrm{CV}=7,63 ; \quad \mathrm{P}<0,0027\right) \quad \mathrm{e}$ $\mathrm{GMD}=0,0854+0,0258 \mathrm{P}-0,00025 \mathrm{P}^{2} \quad\left(\mathrm{r}^{2}=0,3423\right.$; $\mathrm{CV}=21,7 ; \mathrm{P}<0,0029$ ), em que $\mathrm{P}=$ dias em confinamento.

$\mathrm{O} \mathrm{CMS} \%$ apresentou incrementos lineares no decorrer do período experimental, o que indica desenvolvimento volumétrico do rúmen. Os valores observados do CMS\% indicam haver pequeno incremento a partir do terceiro período de avaliação, sobretudo nos níveis de 55 e $65 \%$ de concentrado. Pascoal et al. (2000) observaram que o consumo percentual evoluiu de 2,31 para 2,66 kg/100 kg PV, à medida que o período de avaliação avançou. Verificaram também que o CMS\% estabilizou após 40 dias do início do experimento.
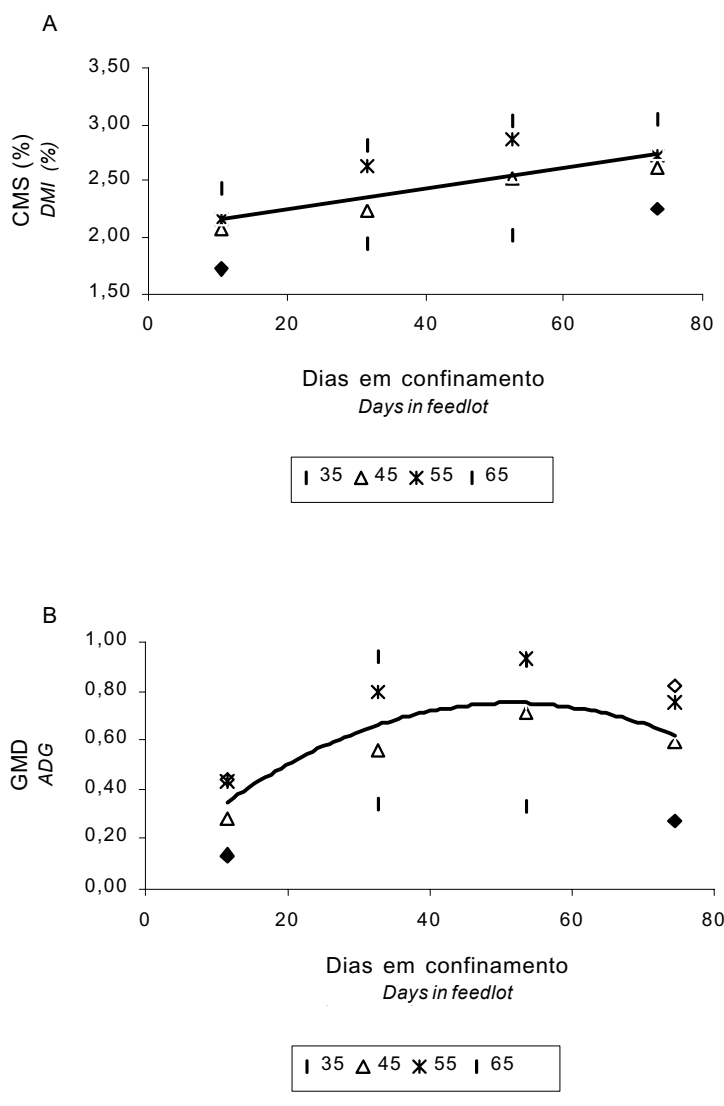

Figura 1 - Evolução do CMS\% (A) e do ganho de peso diário (B), em função do período experimental e dos níveis de concentrado.

Figure 1 - Evolution of the DMI\% (A) and average daily gain $(B)$, according to the experimental period and the concentrate levels.
O GMD apresentou distribuição quadrática com o avanço do período experimental, com pequena redução no último período, possivelmente em decorrência de chuvas e de baixas temperatura, o que aumentou o custo com a energia de mantença. Assim, de acordo com a equação de regressão supracitada, projeta-se ganho máximo de $751 \mathrm{~g} /$ dia aos 52 dias após o desmame.

Embora não tenha sido observada interação significativa entre nível de concentrado e período para CMS\% e GMD, os dados obtidos (Figura 1) indicam maiores valores para os níveis 55 e $65 \%$, sobretudo nos primeiros dias de confinamento. Outros experimentos (Fluharty \& Loerch, 1995) têm sugerido que maiores níveis de concentrado sejam fornecidos nos primeiros dias do confinamento para fornecer a quantidade de energia e proteína suficiente para compensar o baixo consumo inicial e manter a taxa de ganho. Experimentos que avaliam a combinação de níveis de concentrado em diferentes fases de confinamento não tem sido reportados no Brasil com esta categoria animal, enquanto importantes recomendações poderiam ser geradas para esta prática de manejo.

Dessa forma, pode-se recomendar níveis de concentrado acima de $45 \%$ ou a suplementação com pelo menos $1 \%$ PV para adequado desenvolvimento dos bezerros. A observação destes aspectos tem grande importância econômica, uma vez que o concentrado é o mais caro constituinte da dieta (Pascoal et al., 1999). A escolha das dietas para o desmame precoce deve ser criteriosa, posto que esta categoria animal apresenta retorno financeiro mais lento que as categorias em terminação, ainda que a prática do desmame precoce possibilite significativo retorno econômico quando se envolve o gerenciamento completo da propriedade, ou seja, a cria, recria e a terminação.

\section{Conclusões}

Bezerros desmamados precocemente e alimentados com silagem de triticale, associada a maiores quantidades de concentrado na dieta, apresentaram respostas positivas no consumo de matéria seca e no ganho de peso médio diário.

Para manter taxas de ganho de peso condizentes ou superiores àquelas observadas na mesma fase para bezerros desmamados aos 210 dias de idade, o nível mínimo de concentrado na dieta deve ser de $45 \%$, o que equivale à suplementação dos animais com $1 \%$ do peso vivo.

R. Bras. Zootec., v.33, n.6, p.1806-1813, 2004 (Supl. 1) 


\section{Literatura Citada}

ALLEN, M.S. Physical constraints on voluntary intake of forages by ruminants. Journal of Animal Science, v.74, p.3063-3075, 1996.

ANUALPEC. Anuário da pecuária brasileira. São Paulo: O Estado de São Paulo, 2003. 359p.

ARAÚJO, G.G.L.; COELHO DA SILVA, J.F.; VALADARES FILHO, S.C. et al. Consumo e digestibilidade total dos nutrientes de dietas contendo diferentes níveis de volumoso em bezerros. Revista Brasileira de Zootecnia, v.27, n.4, p.998-1005, 1998.

AGRICULTURAL RESEARCH COUNCIL - ARC - The nutrients requirements of ruminants livestock. London: Technical review by on Agricultural Research Council Working Patry, 1980. 351p.

BISHOP, D.K.; WETTERMANN, R.P.; SPICER, L.J. Body energy reserves influenced the onset of luteal activity after early weaning of beef cows. Journal of Animal Science, v.72, p.2703-2708, 1994

CERDÓTES, L.; RESTLE, J.; BRONDANI, I. L. et al. Desempenho produtivo de vacas de quatro grupos genéticos, submetidas a diferentes manejos alimentares desmamadas aos 42 ou 63 dias pós-parto. Revista Brasileira de Zootecnia, v.33, n.3, p.p. 585-596, 2004

COSTA, A.M.; RESTLE, J.; MÜLLER, L. Influência da pastagem cultivada no desempenho reprodutivo de vacas com cria ao pé. Revista do Centro Ciências Rurais, v.11, p.187200, 1981

DESBORDES, C.K.; WELCH, J.G. Influence of specific gravity on rumination and passage of digestibles particles. Journal of Animal Science, v.59, p.470-475, 1984.

EIFERT, E.C.; RESTLE, J.; PASCOAL, L.L. et al. Desempenho de terneiros desmamados precocemente alimentados com silagem de sorgo associada a diferentes níveis de concentrado. In: REUNIÃO ANUAL DA SOCIEDADE BRASILEIRA DE ZOOTECNIA, 36., 1999, Porto Alegre. Anais... Viçosa, MG: Sociedade Brasileira de Zootecnia, 1999 (CD ROM)

FLUHARTY, F.L.; LOERCH, S.C. Effects of protein concentration and protein source on performance of newly arrived feedlot calves. Journal of Animal Science, v.73, p.1585-1594, 1995

MERTENS, D.R. Regulation of forage intake. In: FAHEY JR., G.C. (Ed.). Forage quality, evaluation and utilization. Madison: American Society of Agronomy, 1994. p.450-493.

MOOJEN, J.G., RESTLE, J., MOOJEN, E.L. Efeito da época de desmama e da pastagem no desempenho de vacas e terneiros de corte. 1. Desempenho dos terneiros. Ciência Rural, v.24, n. 12, p.399-403, 1994

MOLETTA, J.L.; PEROTTO, D. Efeito do desmame aos 70 e 210 dias sobre o desempenho ponderal e reprodutivo em vacas de corte. In: REUNIÃO ANUAL DA SOCIEDADE BRASILEIRA DE ZOOTECNIA, 34., 1997, Juiz de Fora, Anais... Juiz de Fora: Sociedade Brasileira de Zootecnia, 1997. p.397-399.

MUEHLMANN, L.D.; ROCHA, M.G.; RESTLE, J.. Utilização de pastagens de estação quente com bovinos desmamados precocemente. Revista Brasileira de Zootecnia, v.26, n.3, p.384-389, 1997

NATIONAL RESEARCH COUNCIL - NRC. Nutrient requirements of beef cattle. 6.ed. Washington, D.C. National Academy Press, 1984. 90p.
NATIONAL RESEARCH COUNCIL - NRC. Nutrient requirements of beef cattle. 7.ed. Washington, D.C.: National Academy Press, 1996. 242p.

NATIONAL RESEARCH COUNCIL - NRC. Nutrient requirements of dairy cattle. 7.ed. Washington, D.C.: National Academy Press, 2001. 381p.

PASCOAL, L.L.; BRONDANI, I.L.; BERNARDES, R.A.C. Avaliação de dietas para desmame, suplementação e confinamento. In: Restle, J. (Ed.). Confinamento, pastagens e suplementação para produção de bovinos de corte. Santa Maria: Universidade Federal de Santa Maria, 1999, p.125-146.

PASCOAL, L.L.; EIFERT, E.C.; RESTLE, J. Níveis de proteína bruta para terneiros desmamados aos 66 dias de idade. Revista Brasileira de Zootecnia, v.29, n.5, p.1537-1544, 2000.

PASCOAL, L.L.; EIFERT, E.C.; RESTLE, J. et al. Efeito de níveis de proteína bruta no desempenho de terneiros de corte desmamados aos 74 dias de idade. In: REUNIÃO ANUAL DA SOCIEDADE BRASILEIRA DE ZOOTECNIA, 38., 2001, Piracicaba. Anais... Piracicaba: Sociedade Brasileira de Zootecnia, CD-ROM. 2001a

PASCOAL, L.L., RESTLE, J., ROSA, J.R.P. et al. Diferentes níveis de concentrado na dieta de bezerros de corte desmamados precocemente. In: REUNIÃO ANUAL DA SOCIEDADE BRASILEIRA DE ZOOTECNIA, 38., 2001, Piracicaba, Anais... Piracicaba: Sociedade Brasileira de Zootecnia, CD-ROM. 2001b.

RESTLE, J., PASCOAL, L.L., ROSA, J.R.P. et al. Influência dos níveis de proteína, via nitrogênio não protéico, no desempenho de bezerros de corte desmamados precocemente. In: REUNIÃO ANUAL DA SOCIEDADE BRASILEIRA DE ZOOTECNIA, 38., 2001, Piracicaba, Anais... Piracicaba: Sociedade Brasileira de Zootecnia, CD-ROM. 2001.

RESTLE, J.; VAZ, F.N. Desmame precoce de terneiros. In: SIMPÓSIO INTERNACIONAL SOBRE PRODUÇÃO INTENSIVA DE GADO DE CORTE, 2., São Paulo. Anais... São Paulo, 1998. p.3-9.

RIBEIRO, E.L.A.; RESTLE, J. Desempenho de terneiros Charolês e Aberdeen Angus puros e seus mestiços com Nelore. Pesquisa Agropecuária Brasileira, v.26, n.8, p.1145-1151, 1991.

ROCHA JR., V.R.; VALADARES FILHO, S.C.; BORGES, A.M. et al. Estimativa do valor energético dos alimentos e validação das equações propostas pelo NRC (2001). Revista Brasileira de Zootecnia, v.32, n.2, p.480-490, 2003.

STATISTICAL ANALYSES SYSTEM - SAS. User's guide. Version 6.0, 4.ed, v.1. Cary: 1990. 890p.

SIMEONE, A.; LOBATO, J.F.P. Efeitos da lotação animal em campo nativo e do controle da amamentação no comportamento reprodutivo de vacas de corte primíparas. Revista Brasileira de Zootecnia, v.25, n.1, p.22-35, 1996.

THOMAS, J.W., TINNIMIT, P. Amounts and sources of protein for dairy calves. Journal of Dairy Science, v.59, n.4, p.1967-1985, 1976.

WELCH, J.G. Rumination, particle size and passage from the rumen. Journal of Animal Science, v.54, p.885-894, 1982.

WILTBANK, J.N.; ROWDEN, W.W.; INGALLS, J.E. et al. Effects of the energy level on reproductive phenoment of nature Hereford cows. Journal of Animal Science, v.21, p.219-225, 1962

Recebido em: 08/09/03 Aceito em: 16/06/04 